\title{
A Sleepy Man with Chronic Obstructive Pulmonary Disease- Obstructive Sleep Apnea Overlap Syndrome
}

\author{
Ki-Hwan Ji \\ Department of Neurology, Busan Paik Hospital, Inje University College of Medicine, Busan, Korea
}

Received November 28, 2017 Revised December 16, 2017

Accepted December 26, 2017

Address for correspondence

Ki-Hwan Ji, MD

Department of Neurology,

Busan Paik Hospital,

Inje University College of

Medicine, 75 Bokji-ro,

Busanjin-gu, Busan 47392, Korea

Tel: +82-51-890-8613

Fax: +82-51-890-6130

E-mail: kihwanji@gmail.com
Chronic obstructive pulmonary disease (COPD) and obstructive sleep apnea (OSA) are prevalent and commonly co-occur as overlap syndrome. Patients with an overlap syndrome have a worse prognosis than those with COPD or OSA alone. Here we report a middle-aged man with overlap syndrome who had multiple comorbidities, managed with continuous positive airway pressure and oxygen therapy.

J Sleep Med 2017;14(2):81-83
Chronic obstructive pulmonary disease (COPD) is common in smoking adult populations and distinctive of airflow limitation not entirely reversible and inflammatory response leading to small airway narrowing and parenchymal destruction of the lungs. ${ }^{1}$ Obstructive sleep apnea (OSA), which is characterized by repetitive partial or complete obstruction of the upper airway, is also prevalent in adults. COPD affects about $10 \%$ and OSA about 3-7\%; COPD and OSA coexist in about $1 \%$ of the adult population. ${ }^{2}$ As COPD and OSA are common, COPD-OSA syndrome has been postulated. ${ }^{3}$ COPDOSA patients have a worse prognosis than those with COPD or OSA alone., ${ }^{2,3}$ Here, we report a middle-aged man with overlap syndrome who had multiple comorbidities such as cor pulmonale, pulmonary hypertension, and one lung ventilation, managed with continuous positive airway pressure (CPAP) and oxygen therapy.

\section{Case Report}

A 43-year-old male visited sleep clinic with complaints of excessive sleepiness and fatigue. He had multiple medical problems: COPD, asthma, and history of pneumonectomy. He

This is an Open Access article distributed under the terms of the Creative Commons Attribution Non-Commercial License (http://creativecommons.org/licenses/by-nc/4.0) which permits unrestricted non-commercial use, distribution, and reproduction in any medium, provided the original work is properly cited. also complained of difficulties in falling asleep and frequent awakenings. He snored loudly and frequently stopped breathing during sleep. Upon awakening, he had a headache with mild to moderate severity. Epworth sleepiness scale score was 19 and STOP-Bang was 5 of 8 (cut-off value of high risky OSA, 3). He denied smoking but drank an average of two bottles of Soju weekly. His medical history revealed his left lung destroyed by tuberculosis and had been surgically removed in his early 20s. He was diagnosed with COPD in his $30 \mathrm{~s}$. Body mass index was $31.02 \mathrm{~kg} / \mathrm{m}^{2}$. Physical examination revealed peripheral edema in four extremities and mild cyanosis on lips. COPD-OSA overlap syndrome was suspected. We consulted with the department of pulmonary medicine and checked baseline workup including chest x-ray, chest CT, transthoracic echocardiogram (TTE) and arterial blood gas analysis (ABGA). ABGA showed pH 7.37, $\mathrm{PaCO}_{2} 63.4 \mathrm{~mm}$ $\mathrm{Hg}, \mathrm{PaO}_{2} 53 \mathrm{~mm} \mathrm{Hg}, \mathrm{HCO}_{3}^{-} 36.2 \mathrm{~mm} \mathrm{Hg}$, and arterial oxygen saturation $85.1 \%$. Chest CT showed compensatory hyperexpansion, multifocal cystic bronchiectasis, and bronchiolitis obliterans in the right lung. TTE revealed pericardial effusion, right ventricular systolic dysfunction, and enlarged size of right atrium and ventricle. Because COPD, cor pulmonale, and pulmonary hypertension were diagnosed, medical treatments including oxygen therapy were started. Polysomnography (PSG) was performed one week later. Because the 


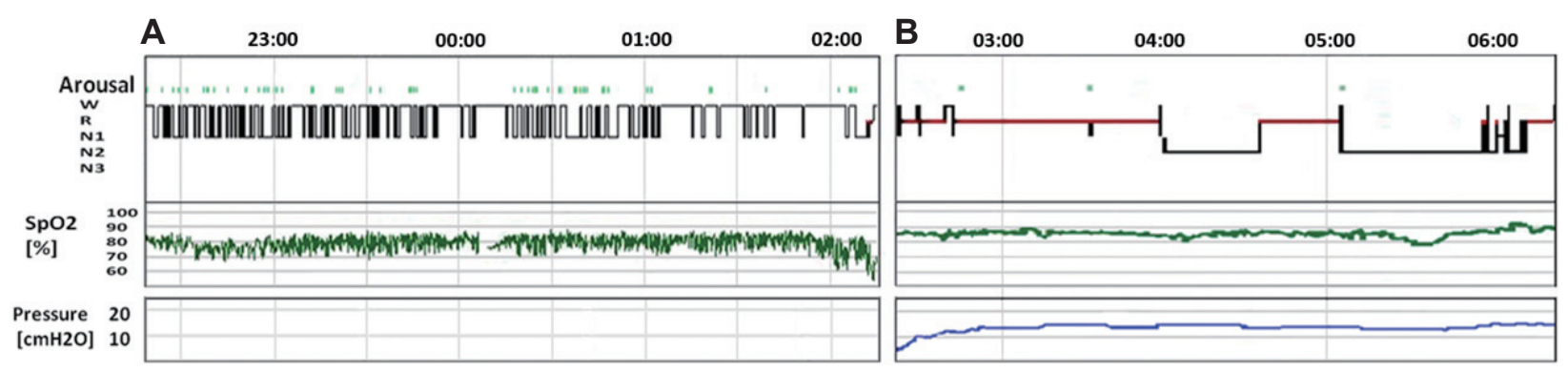

Figure 1. The split-night hypnogram. Hypnogram of diagnostic study $(A)$ shows highly fragmented sleep and intermittent desaturation from a baseline hypoxemia. Hypnogram of titration study (B) shows the large amount of REM sleep, consolidation of sleep and improvement of hypoxemia.

patient had profound oxygen desaturation (average of $74 \%$ ), high apnea-hypopnea index (118/h, 158 events during 80-minute duration of sleep) with fragmented sleep and profound oxygen desaturation (average of 74\%), diagnostic PSG was switched to CPAP. CPAP of $14 \mathrm{cmH}_{2} \mathrm{O}$ and oxygen with $2 \mathrm{~L} /$ min alleviated his sleep symptoms (Fig. 1). After one month, the patient's Epworth sleepiness scale score was 13. One month CPAP data showed a mean apnea-hypopnea index of 3.1/h, percent of CPAP wearing days over $4 \mathrm{~h}$ of $60 \%$, median CPAP usage time of $275 \mathrm{~min} /$ day, and average CPAP usage time of $263 \mathrm{~min} /$ day.

\section{Discussion}

Patients with COPD are classified as two phenotypes: emphysema and chronic bronchitis. ${ }^{4}$ Most patients with COPD have mixtures of emphysema and chronic bronchitis and show a diverse spectrum of clinical phenotypes. Individuals with emphysema phenotype have dyspnea mainly, but their gas exchanges are relatively preserved. To the contrary, patients with chronic bronchitis phenotype have a productive cough and hypoxemia. Our patient was obese and had cor pulmonale. Typically, the chronic bronchitis phenotype patient has higher obesity and has a higher prevalence of cor pulmonale than a typical emphysema phenotype patient. Recent studies have shown that hyperinflation associated with emphysema reduces the risk of OSA, ${ }^{5}$ while chronic bronchitis increases. ${ }^{2}$ The risk of OSA appears to be influenced by spectrums of COPD.

Our patient had a morning headache and showed cyanotic lips and peripheral edema that are common in patient with overlap syndrome. Hypercapnia accounts for his morning headache, and cor pulmonale explains his cyanotic lips and peripheral edema. Cor pulmonale predisposes to OSA because fluid accumulates in the intravascular and interstitial space of the periphery and is redistributed rostrally during sleep in supine position due to gravity. In turn, this influences to upper airway narrowing by accumulation in the neck, increasing tissue pressure. ${ }^{6}$

The mainstay of treatment of COPD patients who show hypoxemia is an oxygen supplementation. ${ }^{3}$ However, in one study looking at the effect of oxygen administration to patients with COPD-OSA overlap syndrome, oxygen therapy improved nocturnal oxygenation but increased the duration of obstructive events. ${ }^{7}$ Oxygen alone should not be installed to patients with COPD at high risk of OSA.

PSG is the most reliable assessment tool for sleep and breathing but also is expensive and time-consuming. Furthermore, PSG might not be accessible to patients. Screening questionnaire such as STOP-Bang may be helpful in the early evaluation of the risk having OSA in COPD patients. ${ }^{2}$ The PSG of our patient showed intermittent oxygen desaturation episodes from baseline hypoxemia, which differ from the typical pattern of intermittent oxygen desaturations from relatively normal baseline saturation in patients with OSA. Baseline hypoxemia is likely a consequence of COPD-OSA overlap having lower baseline oxygen saturation during sleep. ${ }^{8}$

CPAP is standard management option for COPD-OSA overlap syndrome. ${ }^{3}$ COPD-OSA patients not using CPAP have a higher mortality and are more likely to be hospitalized because of severe COPD deterioration, but survival is similar between COPD-OSA receiving CPAP therapy and COPD patients. ${ }^{9,10}$ Consequently, it is crucial to recognize one coexistence of OSA in COPD patients as soon as possible to select and initiate optimal positive airway therapy. In addition, informing diagnosis of COPD to a sleep technician may help CPAP titration focusing on airflow instead of oxygen desaturation. ${ }^{3}$ In case of CPAP failure or COPD dominant overlap syndrome, the appropriate option may be bi-level PAP. ${ }^{2} \mathrm{How}-$ ever, the effects of bi-level PAP on overlap syndrome have not been studied. ${ }^{3}$ Therefore, the choice of non-invasive ventilation for COPD-OSA patients may be different individually, and CPAP should be initially considered.

In this case, CPAP with oxygen therapy was successful. Because there is a high prevalence and poor outcomes associated with this syndrome, COPD patients with high-risk OSA 
should be adequately evaluated and treated.

\section{REFERENCES}

1. Rabe KF, Hurd S, Anzueto A, et al. Global strategy for the diagnosis, management, and prevention of chronic obstructive pulmonary disease: GOLD executive summary. Am J Respir Crit Care Med 2007;176: 532-555.

2. McNicholas WT. COPD-OSA overlap syndrome: evolving evidence regarding epidemiology, clinical consequences, and management. Chest 2017;152:1318-1326.

3. Owens RL, Malhotra A. Sleep-disordered breathing and COPD: the overlap syndrome. Respir Care 2010;55:1333-1344; discussion 13441346.

4. Burrows B, Fletcher CM, Heard BE, Jones NL, Wootliff JS. The emphysematous and bronchial types of chronic airways obstruction. A clinicopathological study of patients in London and Chicago. Lancet 1966; 1:830-835

5. Krachman SL, Tiwari R, Vega ME, et al. Effect of emphysema severity on the apnea-hypopnea index in smokers with obstructive sleep apnea. Ann Am Thorac Soc 2016;13:1129-1135.

6. White LH, Bradley TD. Role of nocturnal rostral fluid shift in the pathogenesis of obstructive and central sleep apnoea. J Physiol 2013; 591:1179-1193.

7. Alford NJ, Fletcher EC, Nickeson D. Acute oxygen in patients with sleep apnea and COPD. Chest 1986;89:30-38.

8. Sanders MH, Newman AB, Haggerty CL, et al. Sleep and sleep-disordered breathing in adults with predominantly mild obstructive airway disease. Am J Respir Crit Care Med 2003;167:7-14.

9. Machado MC, Vollmer WM, Togeiro SM, et al. CPAP and survival in moderate-to-severe obstructive sleep apnoea syndrome and hypoxaemic COPD. Eur Respir J 2010;35:132-137.

10. Marin JM, Soriano JB, Carrizo SJ, Boldova A, Celli BR. Outcomes in patients with chronic obstructive pulmonary disease and obstructive sleep apnea: the overlap syndrome. Am J Respir Crit Care Med 2010; 182:325-331. 